\title{
SOLVABILITY OF QUASILINEAR ELLIPTIC EQUATIONS WITH STRONG DEPENDENCE ON THE GRADIENT
}

\author{
DARKO ŽUBRINIĆ
}

Received 30 May 2000

We study the problem of existence of positive, spherically symmetric strong solutions of quasilinear elliptic equations involving $p$-Laplacian in the ball. We allow simultaneous strong dependence of the right-hand side on both the unknown function and its gradient. The elliptic problem is studied by relating it to the corresponding singular ordinary integro-differential equation. Solvability range is obtained in the form of simple inequalities involving the coefficients describing the problem. We also study a posteriori regularity of solutions. An existence result is formulated for elliptic equations on arbitrary bounded domains in dependence of outer radius of domain.

\section{Introduction}

The aim of this paper is to study existence of weak and strong solutions of the following quasilinear elliptic problem:

$$
\begin{gathered}
-\Delta_{p} u=G(|x|, u,|\nabla u|) \quad \text { in } B \backslash\{0\}, \\
u=0 \quad \text { on } \partial B,
\end{gathered}
$$

$u(x)$ spherically symmetric and decreasing,

where we assume strong dependence on both the unknown and the gradient, see (1.2). Here $B=B_{R}(0)$ is the ball of radius $R$ in $\mathbb{R}^{N}, N \geq 1,1<p<\infty, \Delta_{p} v=$ $\operatorname{div}\left(|\nabla v|^{p-2} \nabla v\right)$ is the $p$-Laplace operator. The Lebesgue measure (volume) of $B$ in $\mathbb{R}^{N}$ is denoted by $|B|$, and the volume of the unit ball is denoted by $C_{N}$. The conjugate exponent of $p$ is defined by $p^{\prime}=p /(p-1)$. Also, we denote $\mathbb{R}_{+}=[0, \infty)$. Weak solution of (1.1) is defined as $u \in W_{0}^{1, p}(B) \cap L^{\infty}(B)$ satisfying (1.1) in the weak sense in $B$. By a strong solution of (1.1) we mean $u \in C^{2}(B \backslash\{0\}) \cap C(\bar{B})$ which satisfies (1.1) pointwise. We also consider weak solutions of quasilinear elliptic equations modelled on general bounded domains. 
This paper represents a continuation of Korkut, Pašić, and Žubrinić [8, 12], where strong dependence on the variable $x$ and on the gradient is allowed on the right-hand side of elliptic equation. Here we allow arbitrary growth rate also in the unknown function. Of course, this requires some additional conditions for solvability, which we find out as a sort of nonresonance conditions.

Similar problems on general bounded domains have been treated by Rakotoson [10], Boccardo, Murat, and Puel [2], Cho and Che [3], Ferone, Posteraro, and Rakotoson [6], see also the references therein. In this paper, we extend the existence result of Rakotoson [10, Theorem 1] to nonlinearities which do not have to satisfy sign condition $\eta \cdot F(x, \eta, \xi) \geq 0$. In Ferone, Posteraro, and Rakotoson [6] the authors consider nonlinearity $F$ which is bounded in the variable $\eta$, while we allow stronger dependence in $\eta$ (however, they allow weaker dependence of $F$ in $x$, see Remark 2.3 below). We also generalize existence result of Cho and Che [3, Theorem 2.2] by allowing more general nonlinearities. Moreover, we allow arbitrary growth rate in the gradient. It is possible to obtain a posteriori regularity of solutions; depending on the value of coefficients, certain solutions may be in $C^{2}(\bar{B} \backslash\{0\}) \cap C^{1}(\bar{B})$, or even in $C^{2}(\bar{B})$, that is, we have classical solutions. In Section 4 , we consider quasilinear elliptic problems in general bounded domains, and formulate existence results which involve geometry of domain together with the structure of the right-hand side.

We impose the following conditions on the right-hand side of (1.1):

$$
\begin{gathered}
0 \leq G(r, \eta, \xi) \leq \tilde{g}_{0} r^{m}+\tilde{h}_{0} \eta^{q}+\tilde{f}_{0} \xi^{e_{0}}, \\
\forall a>0, \quad \exists r \in(0, a), \quad \forall \eta \in \mathbb{R}_{+}, \forall \xi \in \mathbb{R}_{+}, \quad G(r, \eta, \xi)>0 .
\end{gathered}
$$

The first condition in (1.2) is growth condition on the right-hand side of (1.1). We assume that the constants $\tilde{g}_{0}, \tilde{h}_{0}$, and $\tilde{f}_{0}$ are positive real numbers, and $m \leq 0$, that is, the right-hand side of (1.1) may be singular. The role of the second condition in (1.2) is to secure that there is a solution which is positive in $B$. We are interested in finding a solvability range of (1.1), that is, a set of triplets $\left(\tilde{g}_{0}, \tilde{h}_{0}, \tilde{f}_{0}\right)$ such that the corresponding problem (1.1) is solvable.

The main result of this paper is stated in Theorem 2.4(b). As an illustration, we first state its consequence in the case when $p=2, q=1$, and $e_{0}=2$. We consider an elliptic equation with quadratic dependence on the gradient

$$
\begin{gathered}
-\Delta u=\tilde{g}_{0}|x|^{m}+\tilde{h}_{0} \cdot u+\tilde{f}_{0}|\nabla u|^{2} \quad \text { in } B \backslash\{0\}, \\
u=0 \quad \text { on } \partial B,
\end{gathered}
$$

$u(x)$ spherically symmetric and decreasing.

The following corollary of Theorem 2.4(b) shows that the solvability region of elliptic equation (1.3) is related to the spectrum of $-\Delta$, see Remark 1.3.

Corollary 1.1. Let $N \geq 2,-2<m \leq 0$, and

$$
0<\tilde{h}_{0}<\frac{N(m+2)}{R^{2}} .
$$


Assume that $\tilde{g}_{0}$ and $\tilde{f}_{0}$ are positive real numbers such that

$$
\tilde{g}_{0} \cdot \tilde{f}_{0} \leq \frac{(m+N)(2 m+N+2)}{4 R^{m+2}} \cdot\left(1-\frac{R^{2} \tilde{h}_{0}}{N(m+2)}\right)^{2} .
$$

Then (1.3) possesses a positive weak solution $u \in C^{\infty}(\bar{B} \backslash\{0\}) \cap C(\bar{B}) \cap H_{0}^{1}(B)$.

Remark 1.2. If $m=0$, then we can prove existence of a classical positive solution $u \in$ $C^{2}(\bar{B})$ in Corollary 1.1 , see Theorem 3.2. Note that we do not claim that all solutions are classical in this case. The case when $\tilde{h}_{0}=0$ in (1.3) is treated in [8].

Remark 1.3. It is worth noting that condition (1.4) implies that

$$
\tilde{h}_{0}<\lambda_{1}
$$

where $\lambda_{1}$ is the first eigenvalue of $-\Delta$ with zero boundary data. In other words, (1.4) is in fact a nonresonance condition. To show (1.6) recall that

$$
\lambda_{1}=\frac{\mu_{1 N}^{2}}{R^{2}}
$$

where $\mu_{1 N}$ is the first positive zero of the Bessel function of the first kind $J_{N / 2-1}(x)$, see, for example, Dautray and Lions [4, page 747]. Next, for $\mu_{1 N}$ there holds the following inequality:

$$
\mu_{1 N}^{2}>2 N
$$

To see this, we use [11, inequality (1), page 485], which in our notation reads as $\mu_{1 N}>N / 2-1$. This yields $\mu_{1 N}^{2}>(N / 2-1)^{2} \geq 2 N$ for all $N \geq 12$. For $N=1, \ldots, 11$, (1.8) is verified directly using tables of zeros of Bessel functions. Exploiting (1.4) together with (1.7) and (1.8), we obtain (1.6)

$$
\tilde{h}_{0}<\frac{N(m+2)}{R^{2}} \leq \frac{2 N}{R^{2}}<\frac{\mu_{1 N}^{2}}{R^{2}}=\lambda_{1} .
$$

It would be interesting to find solvability conditions for the elliptic problem (1.3) involving arbitrary $\tilde{h}_{0}$. For example, we do not know anything about solvability of (1.3) when $N(m+2) / R^{2} \leq \tilde{h}<\lambda_{1}$.

Remark 1.4. Corollary 1.1 holds also for (1.3) with $g(|x|)$ instead of $\tilde{g}_{0}|x|^{m}$ on the right-hand side, such that $0 \leq g(r) \leq \tilde{g}_{0} r^{m}$, and for any $a>0$ there exists $r \in(0, a)$ such that $g(r)>0$.

\section{A singular ordinary integro-differential equation}

We study solvability of the elliptic problem (1.1) by means of solutions of a suitable singular ordinary integro-differential equation. We follow mainly the approach of [8]. 
162 Solvability of quasilinear elliptic equations

It is convenient to introduce the following constants:

$$
\begin{gathered}
\alpha=p^{\prime}\left(1-\frac{1}{N}\right), \quad \beta=\frac{1}{p-1}, \quad T=|B|, \\
\gamma=1+\frac{m}{N}, \quad \delta=\frac{e_{0}}{p-1}, \quad \varepsilon=\delta\left(1-\frac{1}{N}\right), \\
g_{0}=\frac{\tilde{g}_{0}}{C_{N}^{(m+p) / N} N^{p-1}(m+N)}, \quad h_{0}=\frac{\tilde{h}_{0}}{N^{p} C_{N}^{p / N}}, \quad f_{0}=\frac{\tilde{f}_{0}}{N^{p-e_{0}} C_{N}^{\left(p-e_{0}\right) / N}} .
\end{gathered}
$$

It is possible to obtain existence of strong solutions of (1.1) by studying solutions of the corresponding singular integro-differential equation

$$
\frac{d \omega}{d s}=G^{\omega}(s), \quad s \in(0, T],
$$

where we define

$$
G^{\omega}(s)=\frac{1}{N^{p} C_{N}^{p / N}} G\left(\left(\frac{s}{C_{N}}\right)^{1 / N}, \int_{s}^{T} \frac{\omega(\sigma)^{\beta}}{\sigma^{\alpha}} d \sigma, N C_{N}^{1 / N}\left(\frac{\omega(s)^{\delta}}{s^{\varepsilon}}\right)^{1 / p}\right) .
$$

Note that since we have an integral term, then the mapping $\omega \mapsto G^{\omega}$ is not a Nemytzki operator. We obtain solutions of (2.4) as fixed points of the following nonlinear operator:

$$
\begin{gathered}
K: D(K) \subset C([0, T]) \longrightarrow C([0, T]), \\
K \varphi(t)=\int_{0}^{t} G^{\varphi}(s) d s,
\end{gathered}
$$

with its domain defined by

$$
D(K)=\left\{\varphi \in C([0, T]): 0 \leq \varphi(t) \leq M t^{\gamma}\right\},
$$

where $M>0$ is a constant which does not depend on $\varphi$. Throughout this section we have fixed constants $m, p, N, q, e_{0}, \tilde{f}_{0}, \tilde{g}_{0}$, and $\tilde{h}_{0}$. The corresponding constants $\alpha, \beta$, $\gamma, \delta, \varepsilon$, and $T$ are then defined by (2.1) and (2.2), while $f_{0}, g_{0}$, and $h_{0}$ are defined by (2.3). Once we have a fixed point $\omega$ of $K$, we can generate the corresponding solution of (1.1) using the following lemma. Its proof is analogous to that of Lemma 1 in [8], and therefore we omit it.

Lemma 2.1. Let $\tilde{f}_{0}$ and $\tilde{g}_{0}$ be given positive real numbers. Assume that $1<p<\infty$, $m>-N$, and let condition (1.2) be satisfied. Then for any solution $\omega \in D(K)$ of (2.4) with $T=|B|$, we have that the corresponding function $u(x)$ defined by

$$
u(x)=\int_{C_{N}|x|^{N}}^{|B|} \frac{\omega(t)^{\beta}}{t^{\alpha}} d t, \quad x \in \bar{B},
$$


is a strong solution of the quasilinear problem (1.1). Furthermore, the following relation holds for all $r \in(0, R]$ :

$$
u^{\prime}(r)=-|\nabla u|=-N C_{N}^{1 / N}\left(\frac{\omega(t)}{t^{1-1 / N}}\right)^{1 /(p-1)}, \quad t=C_{N}|x|^{N},
$$

where $u(x)$ is identified with $u(r), r=|x|$.

We deal with strong solutions of (1.1) generated by $\omega \in D(K)$ as described in the above lemma. In the following theorem we say that a function $g: \mathbb{R}^{N} \rightarrow \mathbb{R}$ is nondecreasing if for any $\xi_{1}, \xi_{2} \in \mathbb{R}^{N}$ such that $\xi_{1} \leq \xi_{2}$ componentwise, we have that $g\left(\xi_{1}\right) \leq g\left(\xi_{2}\right)$.

THeOREM 2.2 (existence of solutions). Let $1<p<\infty$,

$$
\max \{-p,-N\}<m \leq 0,
$$

$q>0$, and let $\tilde{g}_{0}, \tilde{h}_{0}$, and $\tilde{f}_{0}$ be positive real numbers. Assume that $G \in C^{k}((0, R] \times$ $\left.\mathbb{R}_{+}^{2}\right)$, where $k \in \varepsilon \mathbb{N} \cup\{0\}$, and let $G$ satisfies conditions (1.2). If $G$ is such that

$$
\exists M>0, \quad g_{0} \leq M-h_{0} \frac{M^{\beta q} T^{q(\beta \gamma-\alpha+1)+1-\gamma}}{(\beta \gamma-\alpha+1)^{q}}-f_{0} \frac{M^{\delta} T^{\gamma(\delta-1)-\varepsilon+1}}{\gamma \delta-\varepsilon+1},
$$

then quasilinear elliptic problem (1.1) possesses at least one strong solution $u \in$ $C^{k+2}(\bar{B} \backslash\{0\}) \cap C(\bar{B})$. If $e_{0}=p$, then $u$ is also the weak solution in $W_{0}^{1, p}(B)$. If $G(r, \eta, \xi)$ is nondecreasing in $\eta$ and $\xi$, then there exists a strong solution which can be obtained constructively using monotone iterations.

Remark 2.3. It is easy to see that condition $-p<m \leq 0$ in Theorem 2.2 implies that

$$
\tilde{g}_{0}|x|^{m} \in L^{s}(B) \quad \forall s>\frac{N}{p},
$$

which appears in [6, page 113]. This shows that our growth condition on the nonlinearity $G$ with respect to $|x|$ is stronger than in [6] (while it is weaker with respect to the unknown and its gradient).

Note that the existence condition (2.11) is fulfilled if the volume $T=|B|$ of the ball is sufficiently small, assuming that the remaining coefficients $m, p, q, N, \tilde{g}_{0}, \tilde{h}_{0}$, and $\tilde{f}_{0}$ are fixed.

Proof of Theorem 2.2. (a) Using Ascoli's theorem we show that the operator $K$ is compact. Note that since $m>-N$, then $\gamma>0$ in (2.7). To prove that the family of functions $R(K)$ is equicontinuous, take any $a, b \in[0, T], a<b$, and $\varphi \in D(K)$. Note that (1.2) implies

$$
\begin{aligned}
\frac{1}{N^{p} C_{N}^{p / N}} \cdot G\left(\left(\frac{s}{C_{N}}\right)^{1 / N}\right. & \left., \int_{s}^{T} \frac{\varphi(\sigma)^{\beta}}{\sigma^{\alpha}} d \sigma, N C_{N}^{1 / N}\left(\frac{\varphi(s)^{\delta}}{s^{\varepsilon}}\right)^{1 / p}\right) \\
& \leq g_{0} \gamma s^{\gamma-1}+h_{0}\left[\int_{s}^{T} \frac{\varphi(\sigma)^{\beta}}{\sigma^{\alpha}} d \sigma\right]^{q}+f_{0} \frac{\varphi(s)^{\delta}}{s^{\varepsilon}}
\end{aligned}
$$


Therefore,

$$
\begin{aligned}
|K \varphi(b)-K \varphi(a)| \leq & g_{0}\left|b^{\gamma}-a^{\gamma}\right|+h_{0} \int_{a}^{b}\left[\int_{s}^{T} \frac{\left(M \sigma^{\gamma}\right)^{\beta}}{\sigma^{\alpha}} d \sigma\right]^{q} d s+f_{0} \int_{a}^{b} \frac{\left(M s^{\gamma}\right)^{\delta}}{s^{\varepsilon}} d s \\
\leq & |b-a|\left(g_{0} \gamma b^{\gamma-1}+\frac{h_{0} M^{\beta q} T^{q(\beta \gamma-\alpha+1)}}{(\beta \gamma-\varepsilon+1)^{q}}\right) \\
& +\frac{f_{0} M^{\delta}}{\gamma \delta-\varepsilon+1}\left[b^{\gamma \delta-\varepsilon+1}-a^{\gamma \delta-\varepsilon+1}\right] .
\end{aligned}
$$

Since $\alpha<\beta \gamma+1$ and $\gamma \delta-\varepsilon+1 \geq \gamma>0$ (which is a consequence of $m>$ $\max \{-p,-N\})$, it follows that the family $R(K)$ is equicontinuous. To show uniform boundedness of the family of functions $R(K)$, we proceed in the similar way:

$$
K \varphi(t) \leq g_{0} t^{\gamma}+\frac{h_{0} M^{\beta q} T^{q(\beta \gamma-\alpha+1)}}{(\beta \gamma-\varepsilon+1)^{q}} \cdot t+\frac{f_{0} M^{\delta}}{\gamma \delta-\varepsilon+1} \cdot t^{\gamma \delta-\varepsilon+1} .
$$

Note that $\gamma \leq 1$ (i.e., $m \leq 0$ ) implies that $t \leq T^{1-\gamma} t^{\gamma}$, and $\gamma \delta-\varepsilon+1 \geq \gamma$ implies $t^{\gamma \delta-\varepsilon+1} \leq T^{\gamma(\delta-1)-\varepsilon+1} t^{\gamma}$. Using this together with (2.11) we conclude that for all $\varphi \in D(K)$,

$$
K \varphi(t) \leq M t^{\gamma}
$$

Therefore, the operator $K$ is compact and $R(K) \subset D(K)$. From Schauder's fixed point theorem we conclude that $K$ possesses at least one fixed point $\omega \in D(K)$. The second condition in (1.2) and $\omega=K \omega$ imply that $\omega(t)$ is increasing on $[0, T]$, and therefore $u$ defined by (2.8) is a decreasing strong solution of (1.1).

Assume that $G$ is of class $C^{1}$, that is, $k=1$. Since a fixed point $\omega$ of $K$ is in $C^{1}((0, T])$, then $K \omega$ is in $C^{2}((0, T])$. Now (2.8) and $\omega=K \omega$ imply that $u \in$ $C^{3}(B \backslash\{0\})$. If $G$ is of class $C^{k}, k \geq 2$, then we proceed in the same way using induction.

The fact that for $e_{0}=p$, the function $u$ is a weak solution of (1.1) contained in $W_{0}^{1, p}(B)$ follows from $m>\max \{-p,-N\}$ in the same way as in the proof of Proposition 11 of [8].

(b) If the function $G(r, \eta, \xi)$ is nondecreasing with respect to $\eta$ and $\xi$, then the operator $K$ is nondecreasing in the sense that if $\varphi \leq \psi$ in $D(K)$ then $K \varphi \leq K \psi$. It is clear that $0 \in D(K)$ is subsolution of $K$, that is, $0 \leq K(0)$ while $\bar{\varphi}(t)=M t^{\gamma}$ is supersolution of $K$, that is, $K \bar{\varphi} \leq \bar{\varphi}$, see (2.16). Since 0 and $\bar{\varphi}$ are ordered subsolution and supersolution and $K$ is compact, the claim follows from Amann [1, Theorem 6.1]: the sequence of monotone iterations $\varphi_{k}$ in $D(K)$ defined by $\varphi_{k}=K \varphi_{k-1}, \varphi_{0}=0$, converges to a fixed point $\omega$ of $K$ in the uniform topology.

We formulate a consequence of Theorem 2.2 in which conditions have more explicit form.

THEOREM 2.4 (existence of solutions). Let $\max \{-p,-N\}<m \leq 0$, and let $\tilde{g}_{0}, \tilde{h}_{0}$, and $\tilde{f}_{0}$ be positive real numbers. Assume that $G \in C^{k}\left([0, R] \times \varepsilon \mathbb{R}_{+}^{2}\right)$, where $k \in \varepsilon \mathbb{N} \cup\{0\}$, and let $G$ satisfy conditions (1.2) and $G(0,0, \xi)>0$ for all $\xi>0$. 
(a) If $q<p-1$ and $e_{0}<p-1$, then (1.1) possesses a strong solution $u \in C^{k+2}(\bar{B} \backslash$ $\{0\}) \cap C(\bar{B})$ for any positive $\tilde{f}_{0}, \tilde{g}_{0}$, and $\tilde{h}_{0}$.

(b) Assume that $e_{0}>q=p-1$, and let $\tilde{f}_{0}, \tilde{g}_{0}$, and $\tilde{h}_{0}$ satisfy the following conditions, see (2.1), (2.2), and (2.3):

$$
h_{0}<\frac{1}{a}, \quad g_{0}^{\delta-1} f_{0} \leq b\left(1-a h_{0}\right)^{\delta}
$$

where we define

$$
a=\frac{T^{q(\beta \gamma-\alpha+1)+1-\gamma}}{\beta \gamma-\alpha+1}, \quad b=\frac{(\delta-1)^{\delta-1}}{\delta^{\delta}} \cdot \frac{\gamma \delta-\varepsilon+1}{T^{\gamma(\delta-1)-\varepsilon+1}} .
$$

Then quasilinear elliptic problem (1.1) possesses at least one strong solution $u \in$ $C^{k+2}(\bar{B} \backslash\{0\}) \cap C(\bar{B})$. If $e_{0}=p$ then the solution is also weak, contained in $W_{0}^{1, p}(B)$.

It is clear that solvability conditions (2.17) have the form

$$
\tilde{h}_{0}<\frac{1}{C_{2}}, \quad \tilde{g}_{0}^{e_{0}\left(p^{\prime}-1\right)-1} \tilde{f}_{0} \leq C_{1} \cdot\left(1-C_{2} \tilde{h}_{0}\right)^{e_{0}\left(p^{\prime}-1\right)},
$$

with explicit positive constants $C_{1}$ and $C_{2}$ depending on $m, N, p, q$, and $e_{0}$. Note that we have $a>0$ and $b>0$ in Theorem 2.4, since inequalities $\beta \gamma-\alpha+1>0$ and $\gamma \delta-\varepsilon+1>0$ follow from $m>-N$ and $m>-p$.

Proof of Theorem 2.4. (a) Since $\beta q<1$ and $\delta<1$, there exists $M_{1}>0$ such that (2.11) holds for all $M>M_{1}$, and the claim follows from Theorem 2.2.

(b) It suffices to show that the envelope of the family of planes in $\mathbb{R}^{3}$ defined by (2.11), parametrized by $M>0$, is the surface defined by (2.17). To this end we have to eliminate $M$ from the system

$$
\begin{gathered}
g_{0}=M-M^{\beta q} A-M^{\delta} B, \\
0=1-\beta q M^{\beta q-1} A-\delta M^{\delta-1} B,
\end{gathered}
$$

where the values of $A$ and $B$ can be easily seen from (2.11). Relation (2.21) is obtained after differentiating (2.20) with respect to $M$. From $\beta q=1$ and $q=p-1$ we easily get $M=((1-A) / \delta B)^{\delta^{\prime}-1}$. Note that we cannot have $B=0$, since this would imply $\tilde{f}_{0}=0$ which is impossible by the first condition in (1.2) and $G(0,0, \xi)>0$ for $\xi>0$. Therefore, system (2.20) and (2.21) yields $g_{0}^{\delta-1} f_{0}=b\left(1-a h_{0}\right)^{\delta}$. Note that the surface $h_{0}=h_{0}\left(g_{0}, f_{0}\right)$ in $\mathbb{R}^{3}$ defined by $h_{0}=(1 / a)\left[1-g_{0}^{1 / \delta^{\prime}}\left(f_{0} / b\right)^{1 / \delta}\right]$ is convex for $g_{0}>0$, $f_{0}>0$, since $d^{2} h_{0}\left(g_{0}, f_{0}\right)>0$. We omit the details.

Proof of Corollary 1.1. Here we use Theorem 2.4(b) together with $p=e_{0}=2, q=1$, and relations (2.1) and (2.2).

Remark 2.5. Similarly as in Remark 1.3, we believe that the constant $C_{2}$ is such that $C_{2}<\lambda_{1}$, where $\lambda_{1}$ is the first eigenvalue of $-\Delta_{p}$. In other words, condition (2.17) seems to be a nonresonance condition, more precisely, it implies $\tilde{h}_{0}<\lambda_{1}$. 
Remark 2.6. Assuming that $\max \{-p,-N\}<m \leq 0, G \in C^{k}\left([0, R] \times \varepsilon \mathbb{R}_{+}^{2}\right), k \in$ $\varepsilon \mathbb{N} \cup\{0\}$, and if $G$ satisfies conditions (1.2), we can also treat some of the remaining cases not covered by Theorem 2.4. Indeed, using (2.11) we easily obtain existence of strong solutions of (1.1) if we assume that $\tilde{g}_{0}, \tilde{h}_{0}$, and $\tilde{f}_{0}$ are positive coefficients such that any of the following three conditions is satisfied:

(i) $q=p-1, e_{0}<p-1, \tilde{g}_{0}>0, \tilde{f}_{0}>0$, and

$$
h_{0}<\frac{(\beta \gamma-\alpha+1)^{q}}{T^{q(\beta \gamma-\alpha+1)+1-\gamma}}
$$

(ii) $q<p-1, e_{0}=p-1, \tilde{g}_{0}>0, \tilde{h}_{0}>0$, and

$$
f_{0}<\frac{\gamma \delta-\varepsilon+1}{T \gamma(\delta-1)-\varepsilon+1}
$$

(iii) $q=p-1, e_{0}=p-1, \tilde{g}_{0}$ is arbitrary and there hold conditions (2.22) and (2.23).

Now we formulate a nonexistence result for quasilinear elliptic equations with strong dependence on the gradient.

Theorem 2.7 (nonexistence). Assume that $m>\max \{-p,-N\}, e_{0}>p-1$ and let the function $G \in C\left([0, R] \times \mathbb{R}_{+}^{2}\right)$ satisfy the condition

$$
G(r, \eta, \xi) \geq \tilde{g}_{0} r^{m}+\tilde{f}_{0} \xi^{e_{0}} .
$$

Let $\tilde{f}_{0}$ and $\tilde{g}_{0}$ be positive numbers such that

$$
g_{0}^{\delta-1} f_{0} \geq \begin{cases}\frac{[\gamma(\delta-1)-\varepsilon+1] \delta^{\delta^{\prime}}}{(\delta-1) T^{\gamma(\delta-1)-\varepsilon+1}} & \text { for } \varepsilon<1, \\ \frac{\gamma \delta^{\delta^{\prime}}}{T^{\gamma(\delta-1)-\varepsilon+1}} & \text { for } \varepsilon \geq 1 .\end{cases}
$$

Then problem (1.1) has no strong solutions. If $e_{0}=p$, then (1.1) has no weak solutions in $W_{0}^{1, p}(B) \cap L^{\infty}(B)$.

This nonexistence result for quasilinear elliptic problem (1.1) is proved analogously as in [12] and therefore we omit it, see also [8]. As we see, if $\tilde{g}_{0}$ and $\tilde{f}_{0}$ are large enough, then condition (2.25) is fulfilled, and there is no strong solution. Since existence and nonexistence regions with respect to $\left(\tilde{g}_{0}, \tilde{h}_{0}, \tilde{f}_{0}\right)$, described by $(2.17)$ and $(2.25)$, are disjoint, we have $b\left(1-a h_{0}\right)^{\delta}<\rho$, where by $\rho$ we denote the right-hand side of (2.25).

Remark 2.8. In Theorem 2.4 we have obtained existence of solutions of (1.1). Since these solutions have integral representation (2.8) with $0 \leq \omega(t) \leq M t^{\gamma}$, it is of interest to know an upper bound of $M$ expressed in terms of the coefficients appearing in elliptic equation (1.1). To this end we use the following elementary lemma, see [13, Lemma 5]. 
Lemma 2.9. Let $c$ and $d$ be positive real numbers and $\delta>1$. Then the condition

$$
\exists M>0, \quad c+d \cdot M^{\delta} \leq M
$$

holds if and only if

$$
c \cdot d^{\delta^{\prime}-1} \leq \frac{\delta-1}{\delta^{\delta^{\prime}}}
$$

Under condition (2.27) property (2.26) is fulfilled with

$$
M_{0}=\left[\frac{c}{d(\delta-1)}\right]^{1 / \delta} .
$$

Assume that conditions of Theorem 2.4(b) are satisfied. By Lemma 2.9, condition (2.17) is equivalent to (2.11). Since $q \beta=1$, from (2.11) we obtain that

$$
\frac{g_{0}}{1-A}+\frac{B}{1-A} \cdot M^{\delta} \leq M
$$

where $A$ and $B$ are from the proof of Theorem 2.4(b). Using Lemma 2.9 again we obtain that we can take

$$
M_{0}=\frac{\delta}{(\delta-1)^{1 / \delta}}\left(\frac{b g_{0}}{f_{0}}\right)^{1 / \delta} .
$$

Note also that we can reprove Theorem 2.4(b) using Lemma 2.9 and Theorem 2.2, since (2.17) follows from (2.27) with $c=g_{0} /(1-A)$ and $d=B /(1-A)$.

Remark 2.10. We have proved the existence of strong solutions of (1.1) in Theorem 2.2 using integral representation (2.8). As we have seen, it is not difficult to see that for $e_{0}=p$ these solutions, as well as the corresponding ones from Theorem 2.4 and in Corollary 1.1, are also weak solutions of (1.1), contained in $W_{0}^{1, p}(B) \cap L^{\infty}(B)$. It suffices to use the same procedure as in Proposition 11 of [8]. However, it is possible to cover the case of general $e_{0}>0$. For example, if in addition to the assumptions of Theorem 2.2 or 2.4 we assume that $e_{0}>0, e_{0} \neq p-1$, and $m>-1-\left(N(p-1) / e_{0}\right)$, then solutions from proofs of Theorems 2.2 and 2.4 are weak. The argument can be seen in Theorem 5 of [12] using obvious modifications.

\section{A posteriori regularity of solutions}

We discuss regularity of solutions that have been obtained in the proof of Theorem 2.2. Note that the following regularity result refers only to solutions of (1.1) that have been obtained by means of integral representation (2.8). That is why we speak about a posteriori regularity. Throughout this section, we assume that the right-hand side of (1.1) has the form

$$
G(|x|, u,|\nabla u|)=\tilde{g}_{0}|x|^{m}+\tilde{h}_{0} \cdot u^{q}+\tilde{f}_{0}|\nabla u|^{p} .
$$

Note that we consider equations with the natural growth in the gradient, that is, $e_{0}=p$. First we study the behaviour of solutions at the origin. 
Lemma 3.1. Let all conditions of Theorem 2.2 be satisfied with (3.1). Let $u$ be a solution of quasilinear elliptic problem (1.1), obtained in the proof of Theorem 2.2 via integral representation (2.8). Then

(a) $u \in C^{\infty}(\bar{B} \backslash\{0\}) \cap C(\bar{B}) \cap W_{0}^{1, p}$ (B) is both weak and strong solution of (1.1).

(b)

$$
\begin{aligned}
\lim _{r \rightarrow 0} \frac{u^{\prime}(r)}{r^{(m+1) /(p-1)}} & =-\left(\frac{\tilde{g}_{0}}{m+N}\right)^{p^{\prime} / p}, \\
\lim _{r \rightarrow 0} \frac{u^{\prime \prime}(r)}{r^{(m-p+2) /(p-1)}} & =-\frac{m+1}{p-1}\left(\frac{\tilde{g}_{0}}{m+N}\right)^{p^{\prime} / p} .
\end{aligned}
$$

Proof. (a) Since $u(x)$ has integral representation (2.8), (1.1) reduces to (see Lemma 2.1):

$$
\frac{d \omega}{d t}=g_{0} \gamma t^{\gamma-1}+h_{0}\left[\int_{t}^{T} \frac{\omega(\sigma)^{\beta}}{\sigma^{\alpha}} d \sigma\right]^{q}+f_{0} \frac{\omega(t)^{\delta}}{t^{\varepsilon}}
$$

where $t=C_{N}|x|^{N}$ and the coefficients are defined by (2.2). This enables to justify the containment $u \in C^{k}(\bar{B} \backslash\{0\})$ inductively with respect to $k$. It is easy to see that (2.8), $0 \leq \omega(t) \leq M t^{\gamma}$, and $m>-p$ imply that $u(0)<\infty$, therefore $u \in C(\bar{B})$. Finally, we can show that $u \in W_{0}^{1, p}(B)$ and that $u$ is a weak solution of (1.1) in the same way as in the proof of Proposition 11 in [8].

(b) Dividing (3.3) by $\gamma t^{\gamma-1}$, we have

$$
\frac{\omega^{\prime}(t)}{\gamma t^{\gamma-1}}=g_{0}+Q_{1}(t)+Q_{2}(t)
$$

where $Q_{1}(t)=h_{0} \gamma^{-1} t^{1-\gamma}\left[\int_{t}^{T}\left(\omega(\sigma)^{\beta} / \sigma^{\alpha}\right) d \sigma\right]^{q}, Q_{2}(t)=f_{0} \gamma^{-1} t^{1-\gamma-\varepsilon} \omega(t)^{\delta}$. We show that there exists $Q_{1}(0):=\lim _{t \rightarrow 0} Q_{1}(t)$, and $Q_{2}(t) \rightarrow 0$ as $t \rightarrow 0$. Using $0 \leq \omega(t) \leq M t^{\gamma}$ and $\beta \gamma-\alpha+1>0$ (which follows from $m>-p$ ) we obtain that

$$
\int_{0}^{T} \frac{\omega(\sigma)^{\beta}}{\sigma^{\alpha}} d \sigma<\infty
$$

From $m \leq 0$ we obtain that $1-\gamma \geq 0$, hence there exists $Q_{1}(0)$. Also, we have $Q_{2}(t) \leq c \cdot t^{\gamma}(\delta-1)-\varepsilon+1 \rightarrow 0$, since the exponent at $t$ is positive, which follows again from $m>-p$. This proves that

$$
\lim _{t \rightarrow 0} \frac{\omega(t)}{t^{\gamma}}=\lim _{t \rightarrow 0} \frac{\omega^{\prime}(t)}{\gamma t^{\gamma-1}}=g_{0}+Q_{1}(0),
$$

where we have used L'Hospital's rule. Now we can proceed in the same way as in the proof of Lemma 8(b) in [8] with $g_{0}+Q_{1}(0)$ instead of $g_{0}$.

An immediate consequence is the following regularity result. 
THEOREM 3.2 (a posteriori regularity). Let all the conditions of Theorem 2.2 be satisfied with (3.1), and let $u$ be a solution of quasilinear elliptic problem (1.1), obtained in the proof of Theorem 2.2 using integral representation (2.8).

(a) If $m<-1$, then $\lim _{r \rightarrow 0} u^{\prime}(r)=-\infty$. In particular, $u \notin C^{1}(\bar{B})$.

(b) If $m=-1$, then

$$
\lim _{r \rightarrow 0} u^{\prime}(r)=-\left(\frac{\tilde{g}_{0}}{m+N}\right)^{p^{\prime} / p} .
$$

As in case (a), we have $u \notin C^{1}(\bar{B})$.

(c) If $-1<m<p-2$, then

$$
\lim _{r \rightarrow 0} u^{\prime}(r)=0, \quad \lim _{r \rightarrow 0} u^{\prime \prime}(r)=-\infty .
$$

In particular, $u \in C^{1}(\bar{B})$ and $u \notin C^{2}(B)$.

(d) If $m \geq p-2$, then $\lim _{r \rightarrow 0} u^{\prime}(r)=0$ and

$$
\lim _{r \rightarrow 0} u^{\prime \prime}(r)= \begin{cases}-\frac{m+1}{p-1}\left(\frac{\tilde{g}_{0}}{m+N}\right)^{p^{\prime} / p} & \text { for } m=p-2, \\ 0 & \text { for } m>p-2 .\end{cases}
$$

In particular, $u$ is a classical solution, $u \in C^{2}(\bar{B})$.

Using lower oscillation estimate from [9] or [7], it is possible to obtain a priori estimate of $u(0)$ from below for any solution of (1.1) obtained in Theorem 2.2. They have precisely the same form as in Proposition 7 of [8], but with $m \leq 0$. We omit the proof.

Proposition 3.3 (estimates of $u(0)$ ). (a) Let $u$ be any solution of quasilinear elliptic equation (1.1) obtained in the proof of Theorem 2.2 with the right-hand side equals to (3.1). Then we have the following a posteriori estimate:

$$
u(0) \leq N \frac{p-1}{m+p} \cdot C_{N}^{(m+p) /(N(p-1))} R^{(m+p) /(p-1)} M_{0}^{p^{\prime}-1},
$$

where $M_{0}$ is defined by (2.30).

(b) For any weak solution $u$ of (1.1) satisfying (3.1), we have the following a priori estimate:

$$
u(0) \geq \begin{cases}\frac{1}{(2 p)^{p^{\prime}}\left(\frac{R^{m+p} \tilde{g}_{0}}{2^{N}-1}\right)^{p^{\prime}-1}} & \text { for } m<0, \\ \left(\frac{c(p, N) R^{p} \tilde{g}_{0}}{p^{p}}\right)^{p^{\prime}-1} & \text { for } m=0,\end{cases}
$$

where

$$
c(p, N)=\sup _{t \in(0,1 / 2)} \frac{t^{p}\left[(1-t)^{N}-t^{N}\right]}{1+t^{N}-(1-t)^{N}} .
$$


In particular, when $p=2, N=2, m=0$, we obtain the following lower bound for weak solutions of (1.1):

$$
u(0) \geq \frac{1}{64} R^{2} \tilde{g}_{0}
$$

\section{Quasilinear elliptic problems on general bounded domains}

It is possible to extend our solvability results for quasilinear equations defined on balls to arbitrary bounded domains $\Omega$ in $\mathbb{R}^{N}$. We consider

$$
-\Delta_{p} u=F(x, u, \nabla u) \quad \text { in } \Omega, \quad u \in W_{0}^{1, p}(\Omega) \cap L^{\infty}(\Omega) .
$$

Here $F \Omega \times \mathbb{R} \times \mathbb{R}^{N} \rightarrow \mathbb{R}$ is a Carathéodory function (i.e., $F(x, \eta, \zeta)$ is measurable with respect to $x$ for fixed $\eta$ and $\zeta$, and continuous with respect to $\eta$ and $\zeta$ for a.e. $x$ ), satisfying the following growth property (note that $e_{0}=p$ ):

$$
-\tilde{g}_{0}\left|x-x_{0}\right|^{m_{0}}-\tilde{h}_{0}|\eta|^{q_{0}}-\tilde{f}_{0}|\zeta|^{p} \leq F(x, \eta, \zeta) \leq \tilde{g}_{1}\left|x-x_{1}\right|^{m_{1}}+\tilde{h}_{1}|\eta|^{q_{1}}+\tilde{f}_{1}|\zeta|^{p},
$$

where $x_{0}$ and $x_{1}$ are given points in $\mathbb{R}^{N}$, and $\tilde{g}_{i}, \tilde{h}_{i}$, and $\tilde{h}_{i}$ are positive coefficients. We introduce constants $\alpha_{i}, \beta_{i}$ analogously as in (2.1), $\gamma_{i}, \delta_{i}, \varepsilon_{i}$ as in (2.2), and $g_{i}, h_{i}, f_{i}$ as in (2.3).

For a given $x_{1} \in \mathbb{R}^{N}$ it is convenient to define outer radius of $\Omega$ with respect to $x_{1}$ :

$$
R\left(x_{1}, \Omega\right)=\max _{x \in \partial \Omega} d\left(x_{1}, x\right) .
$$

In other words, $R\left(x_{1}, \Omega\right)$ is the smallest radius $R$ such that $\Omega$ is contained in the ball $B_{R}\left(x_{1}\right)$. We also denote $T_{1}=\left|B_{R\left(x_{1}, \Omega\right)}\left(x_{1}\right)\right|$. In the following theorem we do not require that either $x_{0}$ or $x_{1}$ be in $\bar{\Omega}$.

We now state an existence result for quasilinear elliptic equations with natural growth in the gradient on general domains. Note that the requirement that $p$ be a quotient of even and odd integers includes the case of $p=2$.

THEOREM 4.1 (existence of solutions). Let $p$ be a quotient of even and odd integers, $1<p<\infty, \max \{-p,-N\}<m_{i} \leq 0, q_{i}>0$, and let $\tilde{f}_{i}, \tilde{g}_{i}, \tilde{h}_{i}$ be positive real numbers, $i=0,1$. Assume that $F[0, R] \times \mathbb{R} \times \mathbb{R}^{N} \rightarrow \mathbb{R}$ is a Carathéodory function satisfying condition (4.2). Assume that the following property holds:

$$
\exists M_{i}>0, \quad g_{i} \leq M_{i}-h_{i} \frac{M_{i}^{\beta_{i} q_{i}} T_{i}^{q_{i}\left(\beta_{i} \gamma_{i}-\alpha_{i}+1\right)+1-\gamma_{i}}}{\beta_{i} \gamma_{i}-\alpha_{i}+1}-f_{i} \frac{M_{i}^{\delta_{i}} T_{i}^{\gamma_{i}\left(\delta_{i}-1\right)-\varepsilon_{i}+1}}{\left(\gamma_{i} \delta_{i}-\varepsilon_{i}+1\right)^{\gamma_{i}}},
$$

for both $i=0,1$. Then quasilinear elliptic problem (4.1) possesses at least one weak solution $u \in W_{0}^{1, p}(\Omega) \cap L^{\infty}(\Omega)$.

Proof. It suffices to find a negative subsolution $\psi_{0}$ and a positive supersolution $\psi_{1}$ in $W_{0}^{1, p}(\Omega) \cap L^{\infty}(\Omega)$, since then we can use [2, Theorem 3.1]. To obtain a negative 
subsolution of (4.1) we consider an auxilliary elliptic equation defined in the ball $B_{0}=B_{R\left(x_{0}, \Omega\right)}\left(x_{0}\right)$ containing $\Omega$ :

$$
\begin{gathered}
-\Delta_{p} u_{0}=-\tilde{g}_{0}\left|x-x_{0}\right|^{m_{0}}-\tilde{h}_{0}\left|u_{0}\right|^{q_{0}}-\tilde{f}_{0}\left|\nabla u_{0}\right|^{p}, \\
u_{0} \in W_{0}^{1, p}\left(B_{0}\right) \cap L^{\infty}\left(B_{0}\right) .
\end{gathered}
$$

We seek a solution $u_{0}(x)$ of this problem in the following form:

$$
u_{0}(x)=\int_{C_{N}\left|x-x_{0}\right|^{N}}^{\left|B_{0}\right|} \frac{\omega(t)^{\beta_{0}}}{t^{\alpha_{0}}} d t
$$

with

$$
\omega \in D_{0}(K)=\left\{\varphi \in C\left(\left[0, T_{0}\right]\right): 0 \geq \varphi(t) \leq-M_{0} t^{\gamma_{0}}\right\},
$$

for some positive constant $M_{0}$ independent of $\varphi$. Therefore, (1.1) reduces to

$$
\frac{d \omega}{d t}=-g_{0} \gamma_{0} t^{\gamma_{0}-1}-h_{0}\left|\int_{t}^{T_{0}} \frac{\omega(\sigma)^{\beta_{0}}}{\sigma^{\alpha_{0}}} d \sigma\right|^{q_{0}}-f_{0} \frac{\omega(t)^{\delta_{0}}}{t^{\varepsilon_{0}}},
$$

with the coefficients defined as in (2.2) and (2.3). Although $\omega(t) \leq 0$ for all $t \in\left[0, T_{0}\right]$, the expression $\omega(t)^{p^{\prime}-1}$ appearing in (4.6) is well defined since $p=2 k /(2 l-1)$, and therefore $u_{0}<0$ in $B_{0}$. Now we proceed similarly as in the proof of Theorem 2.2 to obtain existence of a negative solution $u_{0}$ of (4.1). The desired negative subsolution of (4.1) is then $\psi_{0}=\left.u_{0}\right|_{\Omega}$. Analogously, we find a positive supersolution $\psi_{1}=\left.u_{1}\right|_{\Omega}$ of (4.1), by considering an auxiliary elliptic equation

$$
\begin{gathered}
-\Delta_{p} u_{1}=-\tilde{g}_{1}\left|x-x_{1}\right|^{m_{1}}-\tilde{h}_{1}\left|u_{1}\right|^{q_{1}}-\tilde{f}_{1}\left|\nabla u_{1}\right|^{p}, \\
u_{1} \in W_{0}^{1, p}\left(B_{1}\right) \cap L^{\infty}\left(B_{1}\right),
\end{gathered}
$$

where $B_{1}=B_{R\left(x_{1}, \Omega\right)}\left(x_{1}\right)$.

From Theorem 4.1 we can derive the following result analogously as in the proof of Theorem 2.4.

THEOREM 4.2 (existence of solutions). Let $p$ be a quotient of even and odd integers, $1<p<\infty, \max \{-p,-N\}<m_{i} \leq 0$, and let $\tilde{g}_{i}, \tilde{h}_{i}$, and $\tilde{f}_{i}$ be positive real numbers, $i=1,2$. Assume that $F[0, R] \times \mathbb{R} \times \mathbb{R}^{N} \rightarrow \mathbb{R}$ is a Carathéodory function satisfying condition (4.2).

(a) If $q_{i}<p-1$ and $e_{i}<p-1$, then (4.1) possesses a weak solution $u \in W_{0}^{1, p}(\Omega) \cap$ $L^{\infty}(\Omega)$ for any positive $\tilde{g}_{0}, \tilde{h}_{0}$, and $\tilde{f}_{0}$.

(b) Assume that $e_{i}>q_{i}=p-1$, and let $\tilde{g}_{i}, \tilde{h}_{i}$, and $\tilde{f}_{i}$ satisfy the following conditions:

$$
h_{i}<\frac{1}{a_{i}}, \quad g_{i}^{\delta_{i}-1} f_{i} \leq b_{i}\left(1-a_{i} h_{i}\right)^{\delta_{i}},
$$


172 Solvability of quasilinear elliptic equations

where we define

$$
a_{i}=\frac{T_{i}^{q_{i}\left(\beta_{i} \gamma_{i}-\alpha_{i}+1\right)+1-\gamma_{i}}}{\beta_{i} \gamma_{i}-\alpha_{i}+1}, \quad b_{i}=\frac{\left(\delta_{i}-1\right)^{\delta_{i}-1}}{\delta_{i}^{\delta_{i}}} \cdot \frac{\gamma_{i} \delta_{i}-\varepsilon_{i}+1}{T_{i}^{\gamma_{i}\left(\delta_{i}-1\right)-\varepsilon_{i}+1}} .
$$

Then quasilinear elliptic problem (4.1) possesses at least one weak solution $u \in$ $W_{0}^{1, p}(\Omega) \cap L^{\infty}(\Omega)$.

In the following result we need the notion of outer radius of domain $\Omega$, which is defined by

$$
R(\Omega)=\inf \left\{r>0 \exists x_{1} \in \mathbb{R}^{N}, \Omega \subseteq B_{r}\left(x_{1}\right)\right\} .
$$

Note that the outer radius and diameter of $\Omega$ are related by $R(\Omega) \geq(1 / 2) \operatorname{diam} \Omega$. A solvability result involving outer radius of $\Omega$, with the right-hand side in (1.1) which does not depend on the gradient, can be seen in El Hachimi and Gossez [5].

Corollary 4.3. Let $N \geq 2,-2<m_{i} \leq 0$, and

$$
\tilde{h}_{i}<\frac{N\left(m_{i}+2\right)}{R\left(x_{i}, \Omega\right)^{2}}, \quad i=0,1
$$

where $R\left(x_{i}, \Omega\right)$ is defined by (4.3). Assume that $F[0, R] \times \mathbb{R} \times \mathbb{R}^{N} \rightarrow \mathbb{R}$ is a Carathéodory function satisfying condition (4.2), and let $\tilde{g}_{i}$ and $\tilde{f}_{i}$ be positive real numbers such that

$$
\tilde{g}_{i} \cdot \tilde{f}_{i} \leq \frac{\left(m_{i}+N\right)\left(2 m_{i}+N+2\right)}{4 R\left(x_{i}, \Omega\right)^{m_{i}+2}} \cdot\left(1-\frac{R\left(x_{i}, \Omega\right)^{2} \tilde{h}_{i}}{N\left(m_{i}+2\right)}\right)^{2}, \quad i=0,1 .
$$

Then elliptic problem (4.1) with $p=2$ possesses a weak solution $u \in H_{0}^{1}(\Omega) \cap L^{\infty}(\Omega)$. In particular, if $m_{i}=0$ for $i=0$ or $i=1$, then the corresponding $R\left(x_{i}, \Omega\right)$ can be changed to outer radius $R(\Omega)$ in the above conditions.

Remark 4.4. Note that in Corollary 4.3, condition (4.13) is also a nonresonance condition, since $\Omega \subseteq B_{i}:=B_{R\left(x_{i}, \Omega\right)}\left(x_{i}\right)$ implies that, see (1.9):

$$
\tilde{h}_{i}<\frac{N\left(m_{i}+2\right)}{R_{i}^{2}}<\lambda_{1}\left(B_{i}\right) \leq \lambda_{1}(\Omega),
$$

where $\lambda_{1}(\Omega)$ is the first eigenvalue of the operator $D$ on $\Omega$ with zero boundary data.

\section{Acknowledgement}

I express my gratitude to the referee for his help in the preparation of this paper. 


\section{References}

[1] H. Amann, Fixed point equations and nonlinear eigenvalue problems in ordered Banach spaces, SIAM Rev. 18 (1976), no. 4, 620-709. MR 54\#3519. Zbl 345.47044.

[2] L. Boccardo, F. Murat, and J. P. Puel, Quelques propriétés des opérateurs elliptiques quasi linéaires [Some properties of quasilinear elliptic operators], C. R. Acad. Sci. Paris Sér. I Math. 307 (1988), no. 14, 749-752 (French). MR 90i:35094. Zbl 696.35050.

[3] K. Cho and H. J. Choe, Nonlinear degenerate elliptic partial differential equations with critical growth conditions on the gradient, Proc. Amer. Math. Soc. 123 (1995), no. 12, 3789-3796. MR 96b:35081. Zbl 842.35036.

[4] R. Dautray and J.-L. Lions, Analyse Mathématique et Calcul Numérique pour les Sciences et les Techniques [Mathematical Analysis and Computing for Science and Technology], vol. 2, Masson, Paris, 1987 (French). Zbl 708.35002.

[5] A. El Hachimi and J.-P. Gossez, A note on a nonresonance condition for a quasilinear elliptic problem, Nonlinear Anal. 22 (1994), no. 2, 229-236. MR 94k:35104. Zbl 816.35031.

[6] V. Ferone, M. R. Posteraro, and J. M. Rakotoson, $L^{\infty}$-estimates for nonlinear elliptic problems with p-growth in the gradient, J. Inequal. Appl. 3 (1999), no. 2, 109-125. MR 2001b:35091. Zbl 928.35060.

[7] L. Korkut, M. Pašić, and D. Žubrinić, Control of essential infimum and supremum of solutions of quasilinear elliptic equations, C. R. Acad. Sci. Paris Sér. I Math. 329 (1999), no. 4, 269-274. MR 2000e:35047. Zbl 933.35061.

[8] _ A singular ODE related to quasilinear elliptic equations, Electron. J. Differential Equations (2000), No. 12, 37 pp. (electronic). MR 2000k:35085. Zbl 939.35064.

[9] Some qualitative properities of solutions of quasilinear elliptic equations and applications, J. Differential Equations 170 (2001), 247-280.

[10] J.-M. Rakotoson, Résultats de régularité et d'existence pour certaines équations elliptiques quasi linéaires [Regularity and existence results for some elliptic quasi-linear equations], C. R. Acad. Sci. Paris Sér. I Math. 302 (1986), no. 16, 567-570 (French). MR 87f:35090. Zbl 607.35034.

[11] G. N. Watson, Theory of Bessel Functions, 2nd ed., Cambridge, 1958.

[12] D. Žubrinić, Positive solutions of quasilinear elliptic systems with strong dependence on the gradient, Acta Math. Univ. Comenian. 69 (2000), 183-198.

[13] _ Positive solutions of quasilinear elliptic systems with the natural growth in the gradient, Rend. Istit. Mat. Univ. Trieste 32 (2000), 65-102.

Darko Žubrinić: Department of Applied Mathematics, Faculty of Electrical EngiNEERING AND COMPUTING, Unska 3, 10000 Zagreb, Croatia

E-mail address: darko.zubrinic@fer.hr 


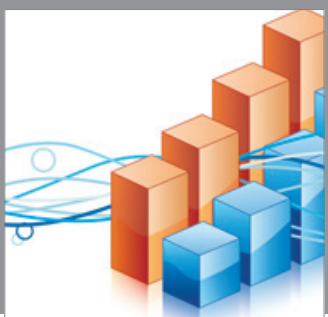

Advances in

Operations Research

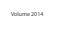

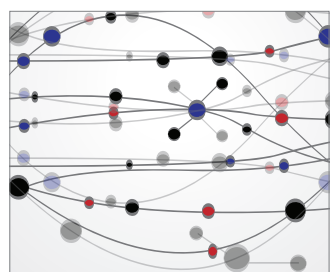

\section{The Scientific} World Journal
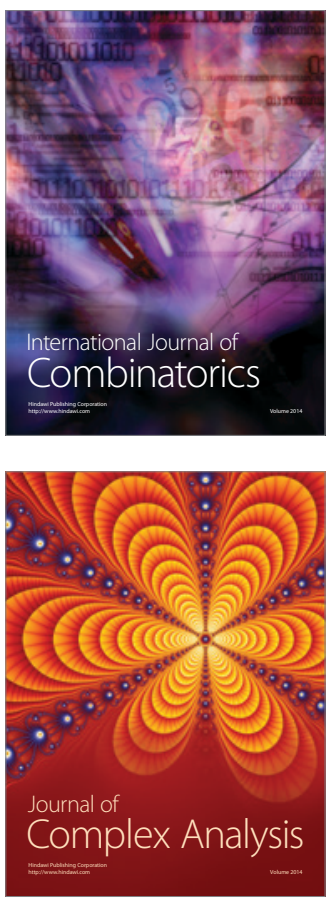

International Journal of

Mathematics and

Mathematical

Sciences
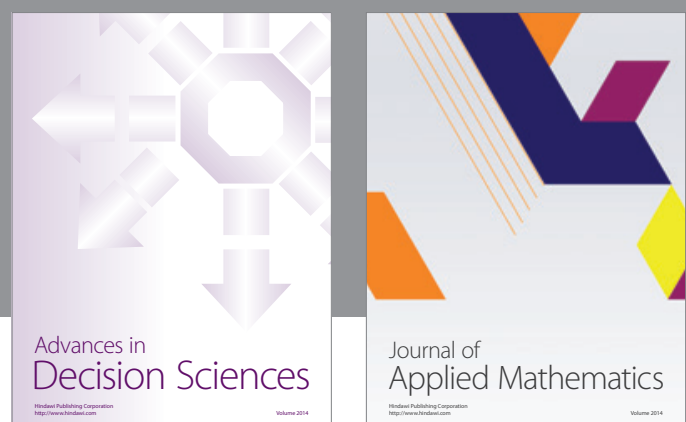

Journal of

Applied Mathematics
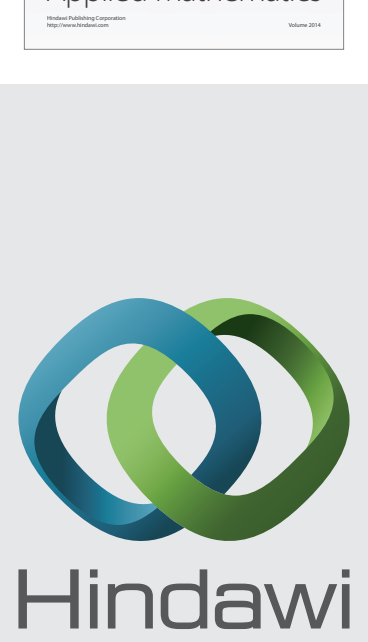

Submit your manuscripts at http://www.hindawi.com
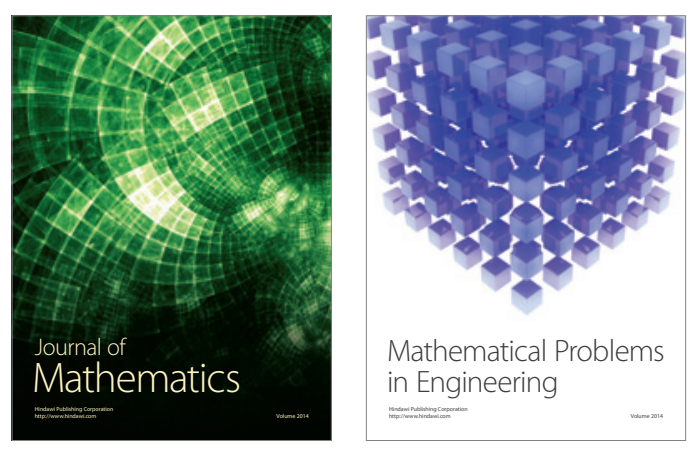

Mathematical Problems in Engineering
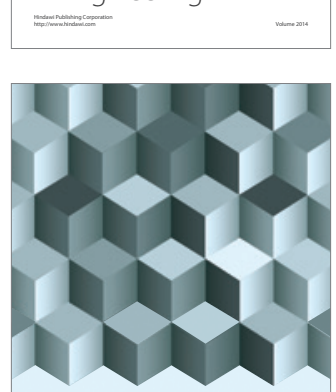

Journal of

Function Spaces
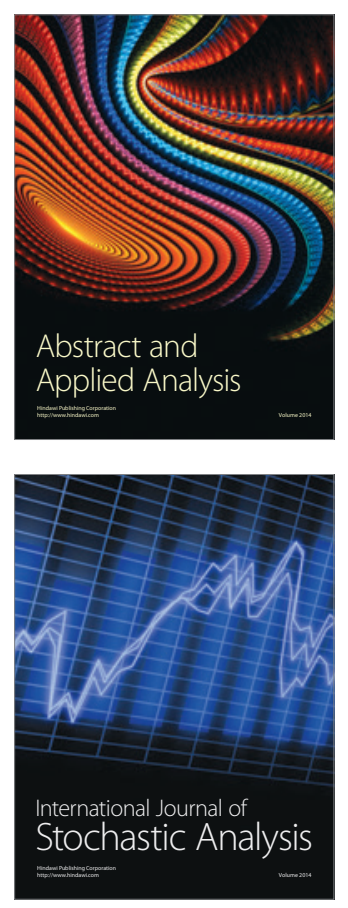

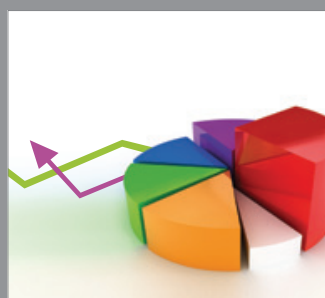

ournal of

Probability and Statistics

Promensencen
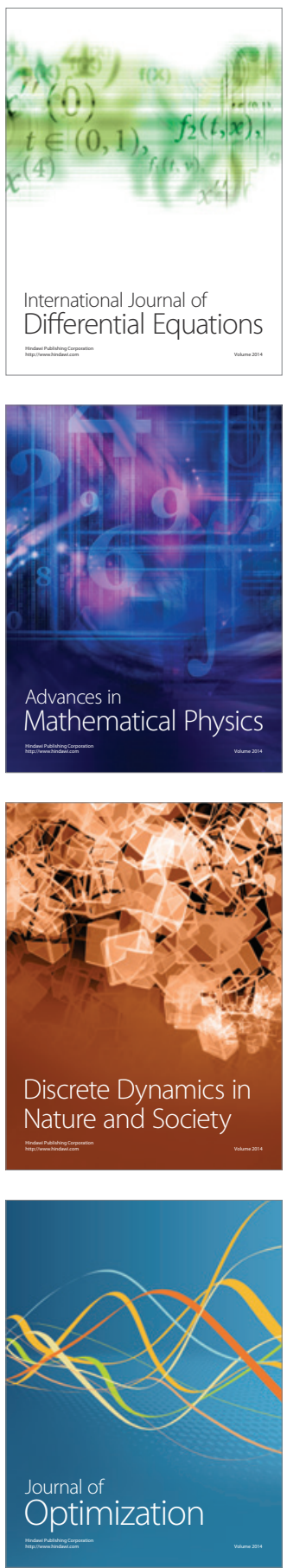